\title{
RINGS OF POLYNOMIALS
}

\section{A. EVYATAR AND A. ZAKS}

Abstract. For an algebra $R$ over a field $k$, with residue field $K$ to be a ring of polynomials in one variable over $k$ it is necessary that $\operatorname{tr} \cdot \operatorname{deg} K / k=1$. We prove that under the hypothesis $\operatorname{tr} \cdot \operatorname{deg} K / k$ $=1, R$ is a ring of Krull-dimension at most one. This is used to derive sufficient conditions for $R$ to be a ring of polynomials in one variable over $k$.

1. Let $k$ be a subfield of the commutative ring $R$. Let $K$ be the quotient field of $R$. The problem we are concerned with is: When is $R$ a ring of polynomials?

In a previous paper [1] we obtained the following result:

If $R$ is a subring of $k\left[x_{1} \cdots x_{n}\right]$ such that with every element of $R$ all of its factors in $k\left[x_{1} \cdots x_{n}\right]$ already lie in $R$, and if $\operatorname{tr} \cdot \operatorname{deg} K / k$ $=n$, then $R$ is a ring of polynomials.

One of the results that we get in this paper is that $R$ is a ring of polynomials also in case $\operatorname{tr} \cdot \operatorname{deg} K / k=1$.

We start by studying rings $R$ for which $\operatorname{tr} \cdot \operatorname{deg} K / k \leqq 1$. We prove that if $R$ is a unique factorization domain, and $R$ is a subring of the ring of polynomials $k\left[x_{1} \cdots x_{n}\right]$, then $R$ is a ring of polynomials.

For subrings of the rings of polynomials over $k$ we prove that

(i) if $R$ is a principal ideal domain then $R$ is a ring of polynomials, and

(ii) if $R$ has a principal ideal $M$ so that $R / M$ is canonically isomorphic to $k$, then $R$ is a ring of polynomials.

Some possible generalizations and modifications are also pointed out.

2. The main object of this section is the study of the rings $R$ for which $\operatorname{tr} \cdot \operatorname{deg} K / k \leqq 1$.

Theorem I. If $k \subset R$, and if $\operatorname{tr} \cdot \operatorname{deg} K / k \leqq 1$, then Krull-dim $R \leqq 1$.

Proof. If $\operatorname{tr} \cdot \operatorname{deg} K / k<1$, then $R$ is a field and the result follows.

Therefore let $\operatorname{tr} \cdot \operatorname{deg} K / k=1$. Assume Krull-dim $R>1$, and let us derive a contradiction. Since there exist prime ideals $P, Q$ in $R$ so that

Received by the editors November 12, 1969.

A MS Subject Classifications. Primary 1393; Secondary 1230, 1420.

Key Words and Phrases. Rings of polynomials, rings of power series, unique factorization domain, principal ideal domain, Euclidean domain, transcendence degree, Krull dimension. 
$0 \neq P \varsubsetneqq Q$, we may choose two elements $p, q$ in $R$ so that $0 \neq p \in P$, $q \in Q$, and $q \notin P$.

Since $0 \neq p \in P, p$ is not algebraic over $k$. Since $\operatorname{tr} \cdot \operatorname{deg} K / k=1$ it follows that there exists an equation

$\left(^{*}\right) \sum_{i, j} k_{i j} p^{i} q^{j}=0, k_{i j}$ in $k$ and not all of them zero.

We may assume that $p$ is not a factor of $\left({ }^{*}\right)$, and this yields an expression $e=\sum_{m} k_{m} q^{m} \neq 0$, with $k_{m} \in k$, such that $e \in P$. Since $e, q$ are elements of $Q$, it follows that $k_{0}=0$. If we presume that $e$ is an expression of smallest possible degree, this leads to a contradiction unless $q \in P$, since $P$ is a prime ideal. But this is a contradiction to the hypothesis $q \notin P$. This proves that Krull-dim $R \ngtr 1$, or Krull$\operatorname{dim} R \leqq 1$ as was asserted.

Remark that if $R$ is a Krull-domain, it follows from the preceding theorem that $R$ is a Dedekind-domain (see [2, p. 24]). If moreover $R$ is a unique factorization domain, then every minimal (=maximal) prime ideal is a principal ideal, and it results that $R$ is a principal ideal domain (see [3, I, p. 244]). Summarizing we have:

Corollary A. Let $R$ a unique factorization domain, $k \subset R$, and $\operatorname{tr} \cdot \operatorname{deg} K / k \leqq 1$. Then $R$ is a principal ideal domain.

3. In this section we will apply the result of $\$ 2$ to subrings of rings of polynomials. The point is that of using induction arguments. We presume for the rest of this section that $R$ is a subring of $k\left[x_{1} \cdots x_{n}\right]$. Recall that the grade of the monomial $x_{1}^{m_{1}} \cdots x_{n}^{m_{n}}$ is $\left(m_{1}, \cdots, m_{n}\right)$. For a polynomial $p$ we set its grade to equal the maximum in the lexicographic order of the grade of its monomials (which has a nonzero coefficient of course), and we denote it by $|p|$. It is easy to verify by straightforward computation that $\left|p_{1} p_{2}\right|=\left|p_{1}\right|+\left|p_{2}\right|$, and that every decreasing sequence of grades $\left|p_{1}\right| \geqq\left|p_{2}\right| \geqq \cdots$ becomes eventually stationary. Finally $|p|=(0, \cdots, 0)$ if and only if $p \in k$.

TheOREM II. Let $R$ be a principal ideal domain, then $R$ is a ring of polynomials over $k$, or else $R=k$.

Proof. If $R=k$ we are done. If not, let $p \in R, p \notin k$ and $p$ of smallest possible grade. Let $q_{1}$ be any other element of $R$ not in $k$. Then for some $a_{1}, b_{1} \in k$, the ideal $I$ generated by $p-a_{1}$ and $q_{1}-b_{1}$ is a proper ideal of $R$ (just take for $a_{1}$ and $b_{1}$ the constant terms of $p$ and $q_{1}$ respectively). Since $R$ is a principal ideal domain we have an element $r$ in $R$ so that $I=R r$. Hence $p-a_{1}=s r$ and $q_{1}-b_{1}=\operatorname{tr}$ for appropriate elements $s, t$ in $R$. Since $|p|=\left|p-a_{1}\right|$, it follows by the minimality of $|p|$ that $|s|=(0, \cdots, 0)$, whence $s \in k$. In particular $s^{-1} \in R$, and therefore $q_{1}-b_{1}=t s^{-1}\left(p-a_{1}\right)$. By the properties of the grade we have (setting $t s^{-1}=q_{2}$ ) 


$$
|q|=\left|q-b_{1}\right|=\left|q_{2}\right|+\left|p-a_{1}\right|>\left|q_{2}\right| \text {. }
$$

In particular, if $q_{2} \notin k$ we repeat the above procedure with $q_{2}$ replacing $q_{1}$. As we obtain this way a strictly decreasing sequence of grades, this procedure must come to an end, namely after a finite number of steps $q_{i} \in k$. We thus get:

$$
\begin{array}{cccc}
q_{1}-b_{1} & =q_{2}\left(p-a_{1}\right) & \text { or } & q_{1}=b_{1}+q_{2}\left(p-a_{1}\right) \\
q_{2}-b_{2} & =q_{3}\left(p-a_{2}\right) & \text { or } & q_{2}=b_{2}+q_{3}\left(p-a_{2}\right) \\
\vdots & \vdots & \vdots \\
q_{i-1}-b_{i-1}=q_{i}\left(p-a_{i}\right) & \text { or } & q_{i-1}=b_{i-1}+q_{i}\left(p-a_{i}\right)
\end{array}
$$

and since $q_{i} \in k$, one obtains by successive substitutions that $q_{j}$ is a polynomial in $p$ for every $j, j=i-1, \cdots, 1$.

We therefore proved that every element in $R$ can be expressed as a polynomial in $p$ with coefficients in $k$. Since $R$ is a domain, and $p$ is not invertible in $R$ it follows that $\sum k_{i} p^{i}=0$ if and only if $k_{i}=0$ for all $i$ 's. Therefore $R$ is a ring of polynomials in one variable over $k$.

Remark that this theorem also tells us that every element of minimal (nonzero) grade can serve as a variable. In view of Corollary A of $\S 2$ we have:

TheOREM III. Let $R$ be a unique factorization domain, and let $\operatorname{tr} \cdot \operatorname{deg} K / k \leqq 1$. Then $R=k$ or else $R$ is a ring of polynomials.

A related problem of interest is: Is a subring $R$ of $k\left[x_{1} \cdots x_{n}\right]$ a ring of polynomials if $R$ is a Dedekind domain?

A case of particular interest is that of factorable rings. Recall that for a ring $R, k \subset R \subset k\left[x_{1} \cdots x_{n}\right]$, to be factorable means that with every element of $R$ all of its factors in $k\left[x_{1} \cdots x_{n}\right]$ already lie in $R$. Since the factorization in a factorable ring is inherited from $k\left[x_{1} \cdots x_{n}\right]$, a factorable ring is necessarily a unique factorization domain. As a consequence we have

Corollary B. If $R$ is a factorable ring and $\operatorname{tr} \cdot \operatorname{deg} K / k=1$, then $R$ is a ring of polynomials over $k$.

Combining this with the result that a factorable ring is a polynomial ring if $\operatorname{tr} \cdot \operatorname{deg} K / k=n$ (see [1]) we have:

THEOREM IV. Every factorable ring in $k\left[x_{1}, x_{2}\right]$ is a ring of polynomials over $k$.

4. In this section we discuss some possible generalizations of Theorem II.

Theorem V. Let $M$ be a principal ideal in $R$, such that $R / M$ is 
canonically isomorphic to $k$. If $R$ is a subring of $k\left[x_{1} \cdots x_{n}\right]$, then $R$ is a ring of polynomials over $k$, or else $R=k$.

Proof. The proof is easily adapted from the proof of Theorem II. The result is obvious if $M=0$. If not, let $p$ be an element in $R$ that is not in $k$, and is of smallest grade. For any element $q$ in $R$ there exists an element $q_{0}$ in $k$ so that $q-q_{0} \in M$. Since $M$ is a principal ideal there exists an $r$ in $R$ so that $M=R r$. Let $q_{1}$ be any element in $R$. There exist $a_{1}$ and $b_{1}$ in $k$ so that $p-a_{1}=s r$ and $q_{1}-b_{1}=t r$ for suitable elements $s, t$ in $R$. We now proceed to complete the proof as in the proof of Theorem II.

REMARK. A closer look at the proof suggests that the condition $R \subset k\left[x_{1} \cdots x_{n}\right]$ is not essential. What is needed for the induction method to work is to have on $R$ a function $f$ into the nonnegative integers such that (i) $f(r)=f\left(r+k_{1}\right)$ for $r \in R-k$ and $k_{1} \in k$ and

(ii) $f\left(r_{1} r_{2}\right)>\max \left(f\left(r_{1}\right), f\left(r_{2}\right)\right)$ for every pair of elements $r_{1}, r_{2}$ in $R$ that are not in $k$.

Another modification is obtained by assuming that on $R$ we have a function $g$ into the nonnegative integers so that $g\left(r_{1}+r_{2}\right) \leqq g\left(r_{1}\right)$ $+g\left(r_{2}\right)$, and $g\left(r_{1} r_{2}\right) \geqq \max \left(g\left(r_{1}\right), g\left(r_{2}\right)\right)$.

A particular case arises if $R$ is a Euclidean domain whose norm satisfies the triangle inequality, and such that the set of elements of minimal norm form a subfield of $R$.

A similar proof applies to the following:

Theorem $\mathrm{V}^{\prime}$. Let $M$ be a principal prime ideal in $R$, let $\mathrm{tr} \cdot \operatorname{deg} K / k$ $\leqq 1$, and let $k$ be algebraically closed. If $R$ is a subring of $k\left[x_{1} \cdots x_{n}\right]$ then $R$ is a ring of polynomials.

We are indebted to Professor D. Zelinsky for suggesting that a similar argument to the one used in the proof of Theorem $\mathrm{V}$ leads to:

Theorem VI. Let $R$ contain a principal ideal $M$ so that $R / M$ is $k$ isomorphic to $k$. If $R$ is complete in the $M$-adic topology then $R$ is either a ring of power series in one variable over $k$, or else $R$ is an Artinian ring, residue ring of a ring of power series in one variable over $k$.

\section{REFERENCES}

1. A. Evyatar and A. Zaks, Purely transcendental subfields of $k\left(x_{1}, \cdots, x_{n}\right)$, Proc. Amer. Math. Soc. 22 (1969), 582-586.

2. P. Samuel, Lectures in commutative algebra, Brandeis University, 1964/65.

3. O. Zariski and P. Samuel, Commutative algebra. Vol. I, The University Series in Higher Math., Van Nostrand, Princeton, N. J., 1958. MR 19, 833.

Technion, Israel Institute of Technology, Haifa, Israel and NorthWestern University, Evanston, IllinoIs 60201 